\title{
Evolution of Incidentally-Discovered Fusiform Aneurysms of the Vertebrobasilar Arterial System: Neuroimaging Features Suggesting Progressive Aneurysm Growth
}

\author{
Toshihiro YASUI, Masaki KOMIYAMA, Yoshiyasu IWAI, Kazuhiro YAMANAKA, \\ Misao NISHIKAWA, and Toshie MORIKAWA
}

Department of Neurosurgery, Osaka City General Hospital, Osaka

\begin{abstract}
This study investigated the natural history and biological behavior of incidental fusiform aneurysms in four patients with incidental fusiform aneurysms of the vertebrobasilar arterial system who had been followed up for more than 3 years (mean 3.5 years). Two lesions remained the same size, and two lesions gradually grew. Angiography showed the non-growing fusiform aneurysms as a circumferentially or unilaterally fusiform dilatation of a short segment of the vertebral artery with smooth walls and a steep slope of the dilatation, and the growing fusiform aneurysms as unilaterally fusiform involving a long segment of the vertebral artery or basilar artery with irregular walls and a gentle slope of dilatation. Magnetic resonance (MR) imaging demonstrated the non-growing fusiform aneurysms as a signal-void area, and the growing fusiform aneurysms as high and intermediate signals in addition to the normal flow void. The heterogeneous MR intensities probably correspond to turbulent flow, laminar flow, thrombosis, or intramural hematoma. Differentiation of growing and non-growing fusiform aneurysms is very difficult at the initial diagnosis. However, enlargement of the fusiform aneurysms is consistent with hemorrhage into the aneurysmal wall, which is confirmed by MR imaging. Fusiform aneurysms with the characteristics of the growing aneurysms cannot be overlooked because of the potential to develop into giant fusiform aneurysms which are very difficult to manage therapeutically.
\end{abstract}

Key words: fusiform aneurysm, dissection, evolution

\section{Introduction}

Fusiform aneurysms of the vertebrobasilar arterial system are difficult to manage therapeutically, especially large and giant fusiform aneurysms. The natural history of intracranial saccular aneurysms is well established, whereas that of fusiform aneurysms is less known. ${ }^{1,13)}$ Therefore, decisions about the management of incidentally-discovered fusiform aneurysms are very difficult. Recently, the number of cases of incidentally-discovered cerebral aneurysms has increased. This follow-up study investigated incidentally-discovered fusiform aneurysms in the vertebrobasilar arterial system to understand the natural history and biological behavior.

\section{Clinical Material and Methods}

Six patients with incidental fusiform aneurysms of the vertebrobasilar arterial system were treated between December 1993 and December 1999. Diagnosis was based on magnetic resonance (MR) imaging and angiography. All fusiform aneurysms were identified incidentally. No patient had any symptoms related to the aneurysms. Four patients with a long-term follow up of more than 3 years were included in this study. The most recent two patients were excluded. Patient characteristics are summarized in Table 1. At the initial admission, both MR imaging and angiography were performed, and then follow-up MR imaging was taken once a year in the outpatient department. In this study, the clinical symptoms and MR imaging findings were retrospectively reviewed with a follow-up period of 38 to 53 months (mean 3.5 years). Blood pressure was controlled in all patients. No antiplatelet agents or anticoagulants were given to any patient. One patient had a clear history of systemic hypertension.

Received May 10, 2001; Accepted August 9, 2001 
Table 1 Clinical characteristics of the four patients with incidental fusiform aneurysms

\begin{tabular}{|c|c|c|c|c|c|c|c|c|}
\hline $\begin{array}{l}\text { Case } \\
\text { No. }\end{array}$ & $\begin{array}{l}\text { Age (yrs) } / \\
\text { Sex }\end{array}$ & $\begin{array}{l}\text { Primary clinical } \\
\text { symptom }\end{array}$ & Site & Side & Past history & $\begin{array}{l}\text { Follow } \\
\text { up (mos) }\end{array}$ & $\begin{array}{l}\text { Change in } \\
\text { aneurysm size }\end{array}$ & $\begin{array}{l}\text { Clinical } \\
\text { course }\end{array}$ \\
\hline 1 & $51 / \mathrm{M}$ & head trauma & VA & $\mathrm{rt}$ & none & 38 & unchanged & asymptomatic \\
\hline 2 & $57 / \mathrm{M}$ & headache & VA & lt & hypertension & 38 & unchanged & asymptomatic \\
\hline 3 & $63 / \mathrm{M}$ & headache & VA & $\mathrm{rt}$ & none & 53 & enlarged & asymptomatic \\
\hline 4 & $64 / \mathrm{M}$ & nasal bleeding & $\mathrm{BA}$ & & $\begin{array}{c}\text { myocardial } \\
\text { infarction }\end{array}$ & 39 & $\begin{array}{l}\text { markedly } \\
\text { enlarged }\end{array}$ & $\begin{array}{l}\text { brainstem } \\
\text { compression }\end{array}$ \\
\hline
\end{tabular}

BA: basilar artery, VA: vertebral artery.

\section{Results}

The four aneurysms could be classified into two groups, based on the changes in aneurysm size. Two patients (Cases 1,2) remained clinically and radiologically silent in the non-growth group. Two patients (Cases 3, 4) showed growth of the aneurysms in the growth group, and Case 4 developed symptoms related to brainstem compression (Table 1).

Angiography showed the aneurysms in the nongrowth group as a circumferentially or unilaterally fusiform dilatation of a short segment of the vertebral artery with smooth walls and a steep slope of the fusiform dilatation (Fig. 1). MR imaging showed no abnormal findings, except dilatation of the signal-void area corresponding to the arterial blood flow (Fig. 2). In contrast, angiography showed aneurysms in the growth group as unilaterally fusiform involving a long segment of the vertebral artery or basilar artery with irregular walls and a rather gentle slope of the fusiform dilatation (Fig. 3). MR imaging showed high and intermediate intensity signals in addition to the normal flow void, suggesting intraaneurysmal turbulent flow, laminar flow, thrombus, or intramural hemorrhage (Fig. 4). Table 2 summarizes these characteristics. Case 3 initially presented with no symptoms, but 2 years after the initial diagnosis, MR imaging revealed slight enlargement of the aneurysm caused by mural hemorrhage occurring in the outermost layer. Thereafter, the aneurysm size remained unchanged. Case 4 is presented in detail below.

\section{Illustrative Case}

Case 4: A 64-year-old man suffered massive nose bleeding. Computed tomography indicated dilatation of the lower basilar artery. Vertebral angiography showed a fusiform dilatation of the lower basilar artery (Fig. 3B). MR imaging showed the lower basilar artery as a fusiform dilatation with a mixture of signal intensities (Fig. 4B). He was followed
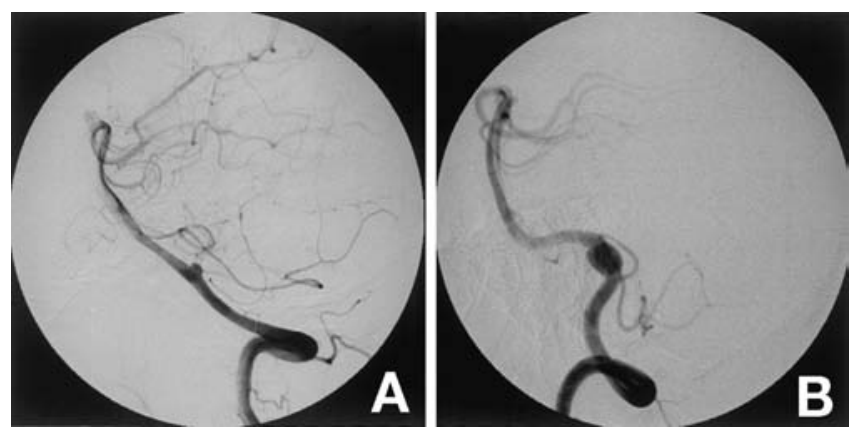

Fig. 1 Vertebral angiograms, lateral view, showing the features of the aneurysms in the nongrowth group. The slope of the fusiform dilatation is rather steep and the walls are smooth. A: Case 1, B: Case 2.

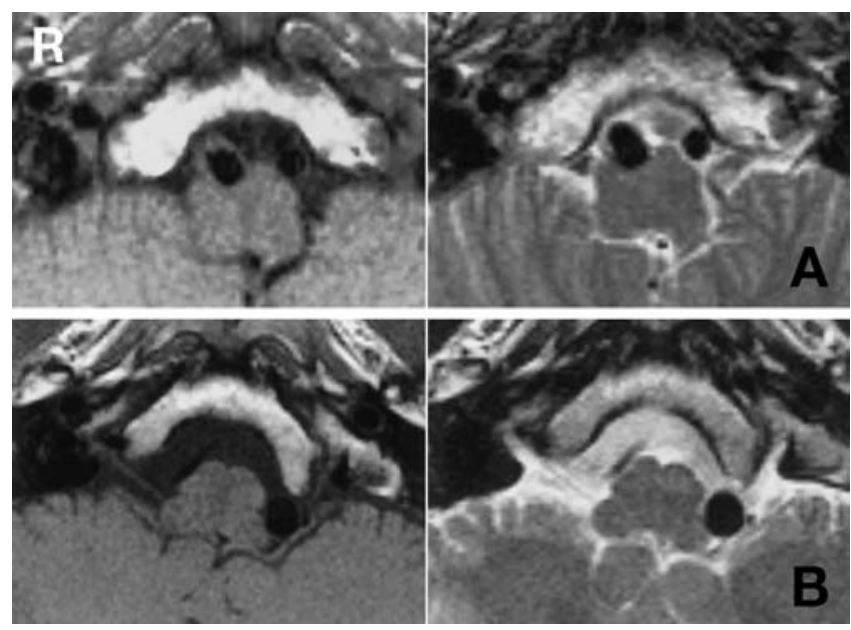

Fig. $2 \mathrm{~T}_{1^{-}}$(left) and $\mathrm{T}_{2}$-weighted (right) magnetic resonance images of the aneurysms in the non-growth group. Only a signal-void area corresponding to the intraaneurysmal blood flow is seen. A: Case 1, B: Case 2.

up by MR imaging in the outpatient department. MR images one year later showed no remarkable 
Table 2 Findings of initial angiography and magnetic resonance (MR) imaging

\begin{tabular}{|c|c|c|c|c|c|}
\hline \multirow{2}{*}{$\begin{array}{l}\text { Case } \\
\text { No. }\end{array}$} & \multicolumn{4}{|c|}{ Angiography } & \multirow[b]{2}{*}{ MR imaging } \\
\hline & Туре & $\begin{array}{l}\text { Involved } \\
\text { segment }\end{array}$ & Wall & Slope & \\
\hline 1 & unilateral & short & smooth & steep & signal void on both $T_{1}$ - and $T_{2}$-weighted images \\
\hline 2 & circumferential & short & smooth & steep & signal void on both $T_{1}$ - and $T_{2}$-weighted images \\
\hline 3 & unilateral & long & irregular & gentle & $\begin{array}{l}\text { mixed intensities on both } \mathrm{T}_{1-} \text { and } \mathrm{T}_{2} \text {-weighted images } \\
\text { (high and relatively high intensities in addition } \\
\text { to signal void area) }\end{array}$ \\
\hline 4 & unilateral & long & irregular & gentle & $\begin{array}{l}\text { mixed intensities on } \mathrm{T}_{1} \text {-weighted image (relatively } \\
\text { high intensity in the signal void area), } \\
\text { signal void on } \mathrm{T}_{2} \text {-weighted image }\end{array}$ \\
\hline
\end{tabular}
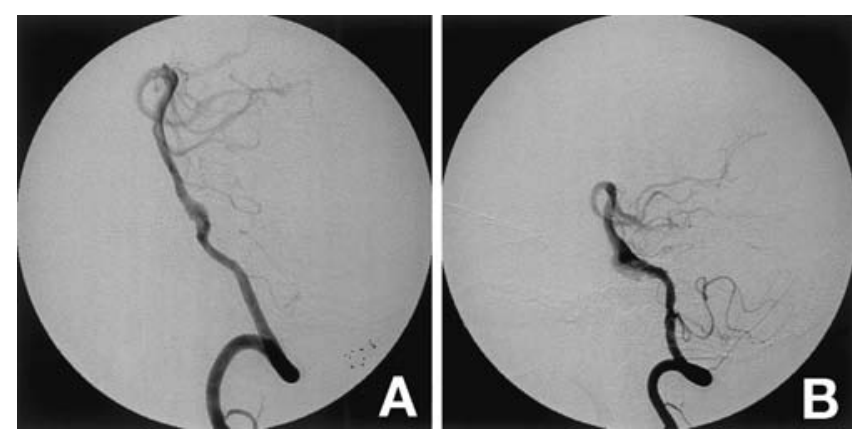

Fig. 3 Vertebral angiograms, lateral view, showing the features of the aneurysms in the growth group. The slope of the fusiform dilatation is gentle and the walls are irregular. A: Case 3, B: Case 4.

changes. Two years later, he did not come to our hospital. Three years later, he suddenly developed vertigo, nausea, and hearing disturbances and was admitted to another hospital. MR imaging performed in that hospital revealed the small fusiform aneurysm had evolved into a giant, serpentine aneurysm (Fig. 5).

\section{Discussion}

Fusiform and dolichoectatic aneurysms occur most frequently in the vertebrobasilar arterial trunk and are among the most difficult aneurysms to treat. There appears to be a spectrum of dolichoectatic aneurysms ranging from a small fusiform aneurysmal dilatation of a single vessel to a giant dolichoectatic aneurysm filled largely with thrombus, also described as a giant serpentine aneurysm. These aneurysms, particularly large partially thrombosed lesions, often cause clinical symptoms due to compression of the surrounding neural structures, ischemia resulting from local perforating vessel thrombo-
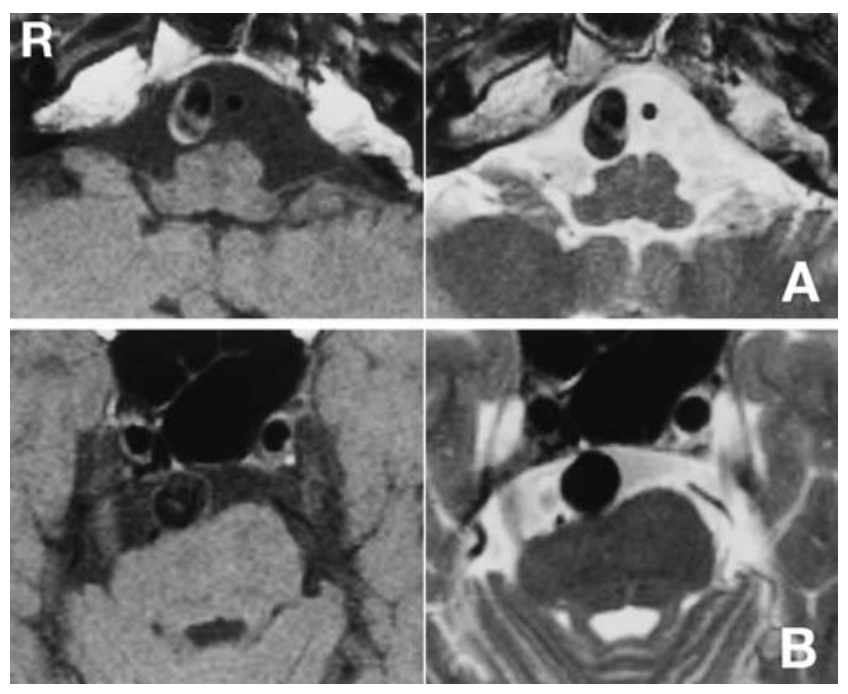

Fig. $4 T_{1^{-}}$(left) and $T_{2}$-weighted (right) magnetic resonance (MR) images of the aneurysms in the growth group. Intraaneurysmal MR intensities are heterogeneous, probably suggesting intramural hematoma. However, turbulent flow, laminar flow, or mural thrombus cannot be excluded. A: Case 3, B: Case 4.

sis or distal thromboembolism, or subarachnoid hemorrhage. ${ }^{1)}$ Symptoms due to brainstem compression are usually slowly progressive, but rapid neurological aggravation can occur although the aneurysm has not ruptured, because of acute swelling of the aneurysm after intramural hemorrhage. ${ }^{15)}$ Some of the small fusiform aneurysms may remain quiescent, but continual growth of a small fusiform aneurysm may lead to the formation of a giant serpentine aneurysm. ${ }^{13)}$ Case 4 is an excellent example of this process.

Defects of the internal elastic laminae are consistent pathological findings in most fusiform 

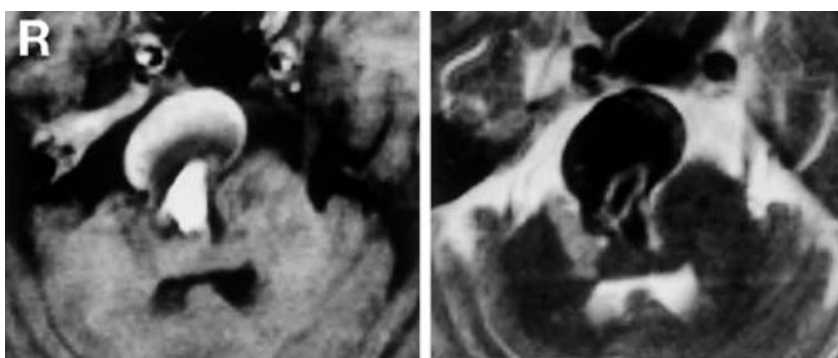

Fig. 5 Case 4. Follow-up $\mathrm{T}_{1^{-}}$(left) and $\mathrm{T}_{2}$-weighted (right) magnetic resonance images at 3 years revealing dramatic growth of the aneurysm and brainstem compression. A newer sickle-shaped clot is located in the periphery of an old thrombus.

aneurysms of the vertebral artery. ${ }^{12,14)}$ The internal elastic lamina contains the elastic tissue and is the most important layer for determining the strength of the vessel wall.6) Therefore, vessels are more prone to damage if elastic tissue is defective. Progressive dilatation of the vessels due to a defect of the internal elastic lamina may be an important factor in the pathogenesis of fusiform aneurysms. ${ }^{7,8,16)}$ Recent findings suggest that the growth of a giant serpentine aneurysm is not always dependent on the pulsatile blood flow and that intramural hemorrhage is a more likely mechanism. Intramural thrombus may be caused by neovascularization and subsequent recurrent hemorrhage within the aneurysmal wall, ${ }^{3,11)}$ or by dissection of the aneurysmal wall due to the residual blood flow in the lumen. ${ }^{4,10)}$

Recently, the clinical presentation of nonatherosclerotic intracranial aneurysms was correlated with the patterns of internal elastic laminae (IEL) disruption. ${ }^{9)}$ Four types were found. Type 1 lesions are classic dissecting aneurysms with a malignant clinical course and show widespread disruption of the IEL without intimal thickening. Type 2 aneurysms are simple ectatic arteries with benign clinical behavior, the IEL is stretched or fragmented, and there is evidence of intimal thickening. Type 3 lesions are dolichoectatic aneurysms, which represent progression of dissection into a serpentine aneurysm, and have fragmented IEL and multiple dissection of thickened intima with luminal thrombus. Type 4 lesions somewhat resemble pure hemodynamic aneurysms not associated with arterial branching and display minimal disruption of the IEL without intimal thickening.9) Probably, our non-growth group corresponds to Type 2, and our growth group to Type 3 in this classification. Differentiation between the growth group and non-growth group of fusiform aneurysms is essential for therapy decision-making and the prognosis, although this may be difficult at the initial diagnosis. Confirmation of the presence of intramural hemorrhage is very important for diagnosis of an aneurysm in the growth group. However, intramural hemorrhage is not always easy to identify, because turbulent flow, laminar flow, or intraaneurysmal thrombus in fusiform aneurysms may mimic the findings of intramural hemorrhage on MR imaging. ${ }^{2)}$ In our present study, fusiform aneurysms in the growth group appeared as various signal intensities in addition to a normal flow void on MR imaging, and had irregular walls, involvement of a long segment, and gently-sloping dilatation on angiography. Recently, another case of enlarging vertebrobasilar dolichoectasia was reported, and the MR imaging and angiography showed the same findings. ${ }^{5)}$ Fusiform aneurysms with these characteristics may have intramural hemorrhage and the potential to cause intramural hemorrhage, and may already be growing and eventually could evolve into a giant, serpentine aneurysm.

\section{References}

1) Anson JA, Lawton MT, Spetzler RF: Characteristics and surgical treatment of dolichoectatic and fusiform aneurysms. J Neurosurg 84: 185-193, 1996

2) Aoki K, Samejima H, Ushikubo Y, Kasai K, Yanai H, Yoshii N: [Seven cases of the fusiform dilatation of the vertebro-basilar system: differential diagnosis by means of CT, MRI, angiography, and autopsy]. CI Kenkyu 14: 545-550, 1992 (Jpn, with Eng abstract)

3) Artmann H, Vonofakos D, Muller H, Grau H: Neuroradiologic and neuropathologic findings with growing giant intracranial aneurysm. Surg Neurol 21: 391-401, 1984

4) Drake CG: Giant intracranial aneurysms: experience with surgical treatment in 174 patients. Clin Neurosurg 26: 12-95, 1979

5) Flemming KD, Josephs K, Wijdicks EFM: Enlarging vertebrobasilar dolichoectasia with subarachnoid hemorrhage heralded by recurrent ischemia. Case illustration. J Neurosurg 92: 504, 2000

6) Glynn LE: Medial defects in the circle of Willis and their relation to aneurysm formation. J Pathol Bacteriol 51: 213-222, 1940

7) Greitz T, Lofstedt S: The relationship between the third ventricle and the basilar artery. Acta Radiol 42: 85-109, 1954

8) Ley A: Compression of the optic nerve by a fusiform aneurysm of the carotid artery. J Neurol Neurosurg Psychiatry 13: 75-86, 1950

9) Mizutani T, Miki Y, Kojima H, Suzuki H: Proposed classification of nonatherosclerotic cerebral fusiform and dissecting aneurysms. Neurosurgery 45: 253-260, 1999 
10) Nagata I, Kikuchi H, Yamagata S, Miyamoto J, Kaneko T, Asato R: [Intraluminal thrombosis and growth-mechanism of giant intracranial aneurysms]. No Shinkei Geka 18: 1115-1120, 1990 (Jpn, with Eng abstract)

11) Schubiger O, Valavanis A, Wichmann W: Growthmechanism of giant intracranial aneurysms; demonstration by CT and MR imaging. Neuroradiology 29: 266-271, 1987

12) Shokunbi MT, Vinters HV, Kaufmann JCE: Fusiform intracranial aneurysms. Clinicopathologic features. Surg Neurol 29: 263-270, 1988

13) Vishteh AG, Spetzler RF: Evolution of a dolichoectatic aneurysm into a giant serpentine aneurysm during long-term follow up. Case illustration. J Neurosurg 91: 346, 1999

14) Yasui T, Komiyama M, Nishikawa M, Nakajima $H$, Kobayashi Y, Inoue T: Fusiform vertebral artery aneurysms as a cause of dissecting aneurysms. J Neu- rosurg 91: 139-144, 1999

15) Yasui T, Yagura H, Komiyama M, Fu Y, Nagata $Y$, Tamura K: [Neurological deterioration induced by giant intracranial aneurysms even though these aneurysms had not ruptured]. No Shinkei Geka 21: 897-901, 1993 (Jpn, with Eng abstract)

16) Yu YL, Moseley IF, Pullicino P, McDonald WI: The clinical picture of ectasia of the intracerebral arteries. J Neurol Neurosurg Psychiatry 45: 29-36, 1982

Address reprint requests to: T. Yasui, M.D., Department of Neurosurgery, Osaka City General Hospital, 2-13-22 Miyakojima-Hondohri, Miyakojima-ku, Osaka 534-0021, Japan.

Commentary on this paper appears on the next page. 


\section{Commentary}

Fusiform aneurysms of the vertebrobasilar arterial system present a significant challenge to neurosurgeons. The natural history of these lesions is poorly understood and therapeutic options are often limited. The authors have made an attempt to study the natural history of these lesions and identify neuroimaging features that predict the natural history. In a very small series of patients, they have suggested that certain features identified on MRI and angiography may predict which lesions are destined to enlarge and become progressively symptomatic and those that will remain quiescent. Perhaps the most revealing difference between these two groups is the MR image. In the non-growth group, there were no abnormal findings other than dilation of the signal void area whereas those that demonstrated future growth had an MRI appearance characterized by high and intermediate intensity signals suggesting turbulent flow.

This is an interesting and thought provoking article that may set the stage for additional studies to further advance our knowledge of the natural history of fusiform aneurysms. Unfortunately, the study only includes four cases with a three-year follow up. Further follow up with many more patients over a longer period of time will be required to verify the importance and accuracy of these findings.

Daniel L. BARROW, M.D. Department of Neurosurgery

The Emory Clinic Atlanta, Georgia, U.S.A.

The authors attempted to differentiate growing fusiform aneurysms from non-growing fusiform aneurysms of the vertebrobasilar artery (VBA) by means of neuroimaging. They suggested that growing aneurysms appeared to involve a long segment of the VBA with irregular wall and gentle slope of dilatation on angiography, and to show heterogeneous MR in- tensities probably due to thrombosis or intramural hematoma. As giant fusiform aneurysms of the VBA have been one of the most formidable intracranial aneurysms, early identification of growing fusiform aneurysms of the VBA is necessary for the practicing cerebrovascular surgeons.

Kyu Chang LEE, M.D. Department of Neurosurgery Yonsei University College of Medicine Seoul, Korea, R.O.K.

Thrombus formation inside a giant or fusiform aneurysm may enhance the production of vascular endothelial growth factor and induce intramural neovascularization. Intramural hemorrhage as a consequence of neovascularization is speculated as the main reason of aneurysmal expansion. In this retrospective study, the authors investigated four patients with fusiform aneurysm of the vertebrobasilar system by annual magnetic resonance imaging (MRI) follow-up. Two patients with suspected intramural thrombosis and hematoma formation manifested as irregular aneurysmal wall on the initial angiography and heterogeneous intensity inside the aneurysm on the initial MRI, subsequently developed aneurysmal growth on the follow-up MRI study. The other two patients showed smooth wall on the initial angiography and only flow void of the aneurysm on the initial MRI did not have the growth of the aneurysm. Thus, the authors suggest that these imaging characteristics may be used for the prediction of the growth of fusiform aneurysm of the vertebrobasilar system. For this conclusion, a prospective study with more case number is needed in the future.

Yong-Kwang TU, M.D. Department of Neurosurgery College of Medicine and Hospital National Taiwan University Taipei, Taiwan, R.O.C. 